\title{
Guidelines and Resources for Prescribing Load using Velocity Based Training
}

Dr Joseph M. Moore BSc (Hons) ${ }^{1} ;$ PhD and Dr Harry F Dorrell BSc (Hons); PhD²

${ }^{1}$ School of Sport, Health and Exercise Science, University of Portsmouth, UK and ${ }^{2}$ School of Sport and Exercise Science, University of Lincoln, UK

\begin{abstract}
Different relative loads during resistance training movements are considered to result in varying physiological adaptions. The challenge facing coaches and athletes is how to determine what absolute load relates to the prescribed relative load and how to account for changes in strength. Velocity based training may offer a solution to this problem. Currently there are limited guidelines for coaches to follow and the proposed methods are associated with increased burden. Therefore, this article provides simple evidence based guidelines and an online application to support the use of velocity based training as a method of auto-regulation during resistance training.
\end{abstract}

\subsection{Introduction}

\subsection{The "problem"}

The use of resistance training to boost athletic performance is widespread within sporting and recreational populations. Regardless of the goals or experience of the participants, the configuration of training variables is a key consideration to maximising the effectiveness of exercise $(8,16)$. Specifically, the number of sets and repetitions, and the prescribed relative load have been shown to be key determinants of the adaptations resulting from resistance training $(21,26)$. The challenge facing strength and conditioning (S\&C) practitioners is how to prescribe and regulate these training variables over training cycles where fluctuations in strength and fatigue mean prescribing a load to elicit certain adaptations is difficult.

Traditionally, loads have been prescribed based on pre-training one repetition maximum (1RM) testing and relative loads aimed at eliciting specific adaptations (e.g. maximal strength $\geq 85 \% 1 \mathrm{RM})(22,24)$. To account for changes in strength due to muscular adaptations, standardized increases in prescribed loads (e.g. week 1: $80 \%$ 1RM; week 2: 82\% 1RM) are often programmed. Other approaches include auto-regulatory methods where load is manipulated using ratings of perceived exertion (RPE) or repetitions in reserve (RIR) $(9,14,27)$. Such methods allow practitioners to progress or regress resistance programmes to maximise their effectiveness. Despite their widespread use, and identified effectiveness (13), the use of programmed systematic increases in load and subjective measures of athlete perception have associated limitations (4, 15, 25). 
Prescription of load based on 1RMs is susceptible to errors due to the day-to-day variation in absolute strength that can be as high as $18 \%$ (15), and gains in strength that will cause the pre training assessments to no longer represent the true strength of an athlete (15). These limitations may result in sub-optimal load prescription, potentially compromising the intended training stimulus and subsequent physiological adaptations. Auto-regulation methods, such as using RPE to manipulate load, may address these issues but are associated with their own limitations. Altering load based on subjective athlete feedback may lead to between session and athlete inconsistencies due to training experience, daily motivation, and current perceived fatigue $(11,12)$. Additionally, the accuracy of an athlete's perceived and actual RIR has been shown to reduce the further from failure they are, limiting the efficacy of such approaches when lifting sub-maximal loads $(11,27)$. The limitations to the currently employed methods of achieving optimal load prescription mean that despite their widespread use, they may fail account for fluctuations in athlete strength and fatigue that occur on a set by set and session by session basis. One area that may provide a more effective approach is velocity based training (VBT).

\subsection{Velocity Based Training}

The use of lift velocity within S\&C practices has grown since the ability to accurately measure such variables has become more accessible with tools ranging from linear positional transducers (6) to mobile applications (2). The velocity that the concentric section of a lift is performed has been shown to be related to its relative load $(10,19)$. This relationship between concentric lift velocity and relative load is referred to as a load-velocity profile (LVP;
Figure 1). LVPs have been shown to remain unchanged despite significant increases in absolute strength $(1,10)$, and have therefore been theorised as a potential auto-regulatory approach for prescribing training load. Early explorations into LVPs focussed on developing group equations that could be implemented into other samples of a similar training demographic $(2,19)$, these data provided practitioners with average profiles. Recent research has begun to highlight that LVPs are individual to each athlete and therefore that group profiles although being accurate for some athletes (1), may not be for others (Figure 1 ). The positives and negatives of using group and individual profiles are discussed later in the article however, both can be used as a method of auto-regulation $(5,7)$.

The theorised benefit of the use of an LVP to inform training prescription is based on the assumption that despite changes in absolute strength, due to training stimuli or fatigue, the profile will enable a practitioner to estimate the relative load being lifted by measuring the velocity of the lift (15). By estimating the percentage of $1 \mathrm{RM}$ a load represents, the athlete's acute 1RM (15) and subsequently the load relating to a certain percentage (7) can be calculated. This approach provides practitioners with a method by which setby-set changes can be accounted for, ensuring athletes are lifting at a set percentage of their acute 1RM. In comparison to other auto regulation methods, VBT may therefore offer an objective approach that is able to adjust load to ensure that athletes are lifting the correct load that has been programmed to elicit specific physiological adaptations. One cautionary note on the use of LVPs to predict an athlete's $1 R M$, is that current evidence suggests it is associated with small errors $(3,18)$, and therefore the 
proposed method is not fully supported as an approach for athlete testing. Instead the errors of using an LVP to predict an athlete's current strength may still allow a more accurate dictation of training load than other auto-regulatory methods.

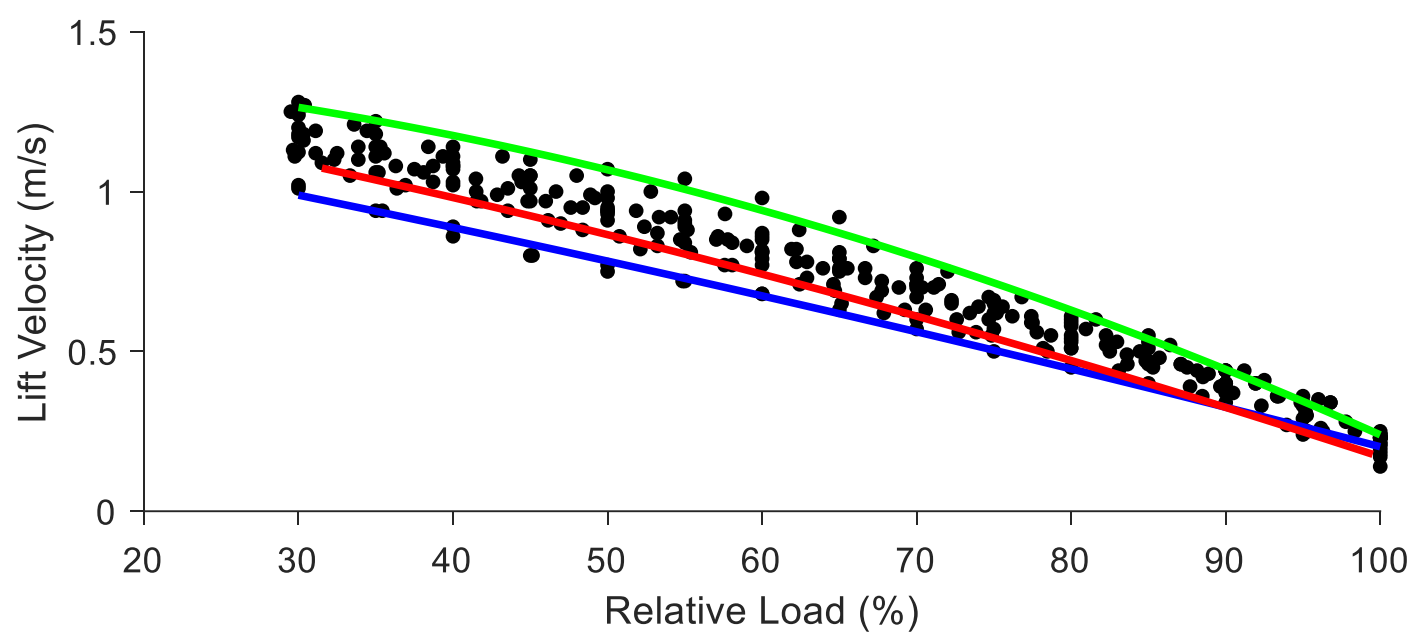

Figure 1. Velocity data from a group of resistance trained males $(n=19)$ and the load-velocity profiles of three individuals within the sample highlighting the differences in profiles of different athletes. Data used with permission from Dorrell, Moore and Gee (5).

Few studies have explored the validity of a VBT approach to auto-regulation within training cycles however, those that have appear to support its benefits $(4,5,7)$. Dorrell, Smith and Gee (7) compared traditional load prescription based on pretraining 1 RMs to a VBT approach using group LVPs to dictate load on a set-by-set basis in 16 resistance-trained men (Table 1). Over a six-week strength and power programme, significant increases $(p<0.05)$ in maximal strength for back squat (VBT $9 \%$, PBT $8 \%$ ), bench press (VBT 8\%, PBT $4 \%$ ), strict overhead press (VBT 6\%, percentage based training $6 \%$ ), and deadlift (VBT 6\%) were identified. In addition to the data supporting similar or favourable adaptations when using VBT, these improvements were achieved despite a significantly lower total volume lifted in the back squat (9\%), bench press (6\%), and strict overhead press (6\%). Similar or greater adaptations despite less total volume supports using group LVPs to dictate load. The use of group profiles within this study supports that despite their associated errors they still provide a usable and effective tool for manipulating training load. Despite this, individual profiles may provide a more effective and accurate approach to auto-regulation than group profiles.

Dorrell, Moore and Gee (5) compared the adaptations to a six-week strength and power resistance program in 19 trained males (Table 1) when regulating load using either a group or individual LVP. Both interventions were shown to significantly ( $p<0.05$ ) increase squat 1RM with individual LVPs resulting in greater gains (individual: 9.7\%; group: $7.2 \%$ ). The data provides further support for the use of LVPs in resistance-training regulation, and supports that errors associated with group profiles make them less effective compared to an individual approach when applied in this manner. Individual profiles although appearing to be more effective are associated with increased testing and athlete burden, and therefore may not be viable in certain situations. 
The discussed use of VBT for regulating and prescribing training load offers S\&C practitioners a potential solution for how to maximise programmed resistance training activities whilst avoiding some of the limitations related to currently employed auto-regulation methods. However, VBT is associated with its own limitations, specifically in relation to its application to professional and applied settings. Firstly, the collection of individual LVPs requires athletes to work to their $1 R M$, something that is not viable within some populations; and secondly, once an LVP is obtained a number of computational steps are required each time it is used to calculate the required load. Therefore, the aim of this article is to provide guidelines for the use of VBT to dictate training load, and provide a free online application to enable this to be implemented into applied S\&C practice.

\subsection{Proposed Methods for using VBT to Dictate Load}

There are two steps to implementing VBT as a method of dictating load, obtaining, and using the LVP. Both of these steps are described in detail below, including background information and practical guidelines. A flow diagram is presented in Figure 2 to provide a systematic guide to using VBT to dictate load, and highlight certain sections of the article that are useful depending on the intended use.

\subsection{Athlete Profiling}

\subsubsection{Background}

The foundation of using VBT to dictate load is the $\operatorname{LVP}(15,19)$. Recent evidence has supported that each athlete has a unique $\operatorname{LVP}(4,5)$ and that despite changes in absolute strength the profile acts as a passport of sorts that describes an athlete's ability to lift a percentage of their maximum at a set velocity. More research is required to explore whether periods of training and detraining (e.g. injury layoffs) result in changes in this profile and for how long this profile remains accurate. What evidence is currently available supports that periods ( $\sim 6$ weeks) of strength training, despite increases in absolute strength (mean $\pm S D ; 9.3 \pm 6.7 \%$ ), do not result in changes in the LVP (10). As described, group velocity profiles from published literature or squad testing offer a general LVP that can be used as a mode of auto regulation when prescribing training variables, however to maximise the effectiveness and where the environment permits, individual athlete profiling should be conducted. It should be noted that each movement has its own LVP, and therefore requires separate profiling for each athlete or group (20).

The method by which the LVP of a given movement is obtained has yet to be explored within the literature, resulting in a variety of approaches being employed. Although attempts have been made to obtain an LVP through submaximal testing the associated errors relating to prediction of a $1 \mathrm{RM}$ and the velocity at which that maximal lift would be completed mean it is not a suitable approach (17). The generally accepted method creates the LVP retrospectively from a selection of maximal effort lifts at varying loads. Participants complete a given number of repetitions (inversely related to mean velocity or relative intensity) at a range of increasing loads, working to a $1 \mathrm{RM}$. Once all data has been collected, relative load and the respective velocity output (generally mean concentric or mean propulsive) are plotted, before a line of best fit is fitted. Two considerations should be accounted for 1) the accuracy of an LVP increases as more relative loads are tested, 
and 2) the measured velocities should be of unfatigued lifts and therefore sets should be separate by adequate rest and consist of only 1-3 repetitions.

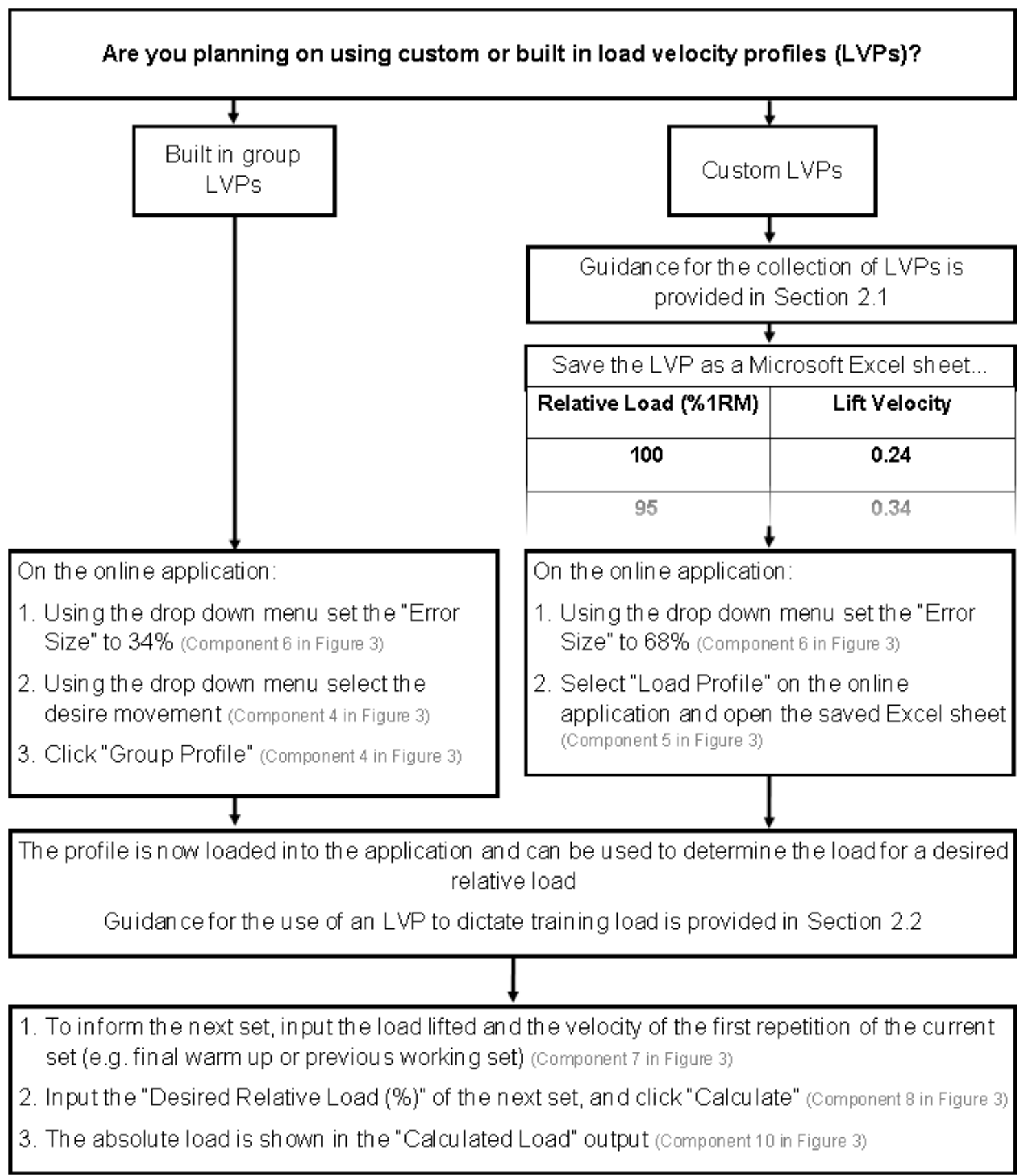

Figure 2. A flow diagram of the steps to using a load velocity profile, lift velocity, and the online application to dictate load during resistance training exercises.

A link to a free online application (MATLAB WebApps, The MathWorks Inc., Natick, MA, US) is included in this article (Figure 3 ). The application is capable of calculating an LVP from velocity data, and using this LVP to provide estimates of an athlete's 1RM. Group LVPs for resistance-trained males are included for squat, deadlift, and strict overhead press taken from the authors (HD) research $(5,7)$. This application is 
compatible with laptop browsers and tablets, and can be accessed through the following URL (https://matlabwebapps.port.ac.uk/webapps/home/).

The application intends to increase the accessibility of the method of dictating load described in this article for practitioners and researchers. The application intends to increase the accessibility of the method of dictating load described in this article for practitioners and researchers. Due to limitations with the host server, the application has a maximum number of concurrent users and therefore a Microsoft Excel Document is provided (Supplemental Data File 1) that can be used with the included group LVPs. Please cite this article when using the application or spreadsheet for research purposes. 


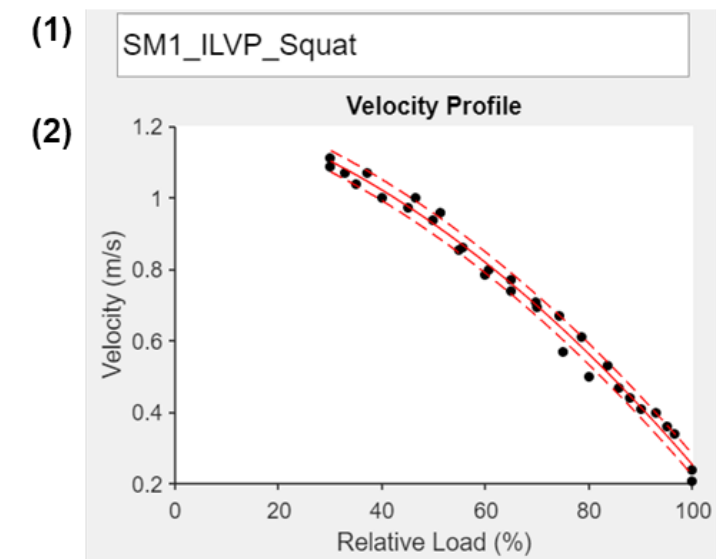

(3)

(4) Group Profile Squat

(5) Load Profile

(6) Error Size $68 \%$ $~ M a n u a l$ Error 0.0296

\begin{tabular}{|c|c|c|c|}
\hline \multicolumn{4}{|c|}{ Inputs } \\
\hline \multicolumn{4}{|l|}{ Current Set } \\
\hline Load lifted (kg/lbs) & 150 & Velocity $(\mathrm{m} / \mathrm{s})$ & 0.6 \\
\hline \multicolumn{4}{|l|}{ Next Set } \\
\hline \multicolumn{2}{|c|}{ Desired Relative Load (\%) } & \multicolumn{2}{|l|}{85} \\
\hline \multicolumn{4}{|c|}{ Calculate } \\
\hline \multicolumn{4}{|c|}{ Outputs } \\
\hline \multicolumn{2}{|c|}{ Calculated Load (kg/lb) } & \multicolumn{2}{|c|}{ Estimated 1RM (kg/lb) } \\
\hline \multicolumn{2}{|c|}{160.8} & & 89.2 \\
\hline \multicolumn{4}{|c|}{ Velocity Details } \\
\hline Upper 0.52 & \multirow{2}{*}{\multicolumn{2}{|c|}{ Velocity Stop (\%) $20 \quad \mathbf{v}$}} & \\
\hline \multirow{2}{*}{\multicolumn{2}{|c|}{$\begin{array}{ll}\text { Target } & 0.49 \\
& 0.3902 \mathrm{~m} / \mathrm{s}\end{array}$}} & & \\
\hline & $0.3902 \mathrm{~m} / \mathrm{s}$ & & \\
\hline \multicolumn{3}{|c|}{ 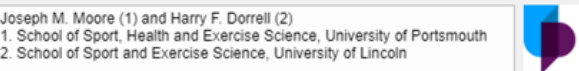 } & 站 \\
\hline
\end{tabular}

\section{1) LVP name}

2) Graphical presentation of the LVP

3) Equation of the LVP $\left(y=a x^{2}+b x+c\right)$

4) Button and menu to load included group LVP

(8) 5) Import velocity data from an excel document

(9)

6) The level of error to include in the LVP

7) Data inputs of the lifted load and velocity

(10)

8) The relative load of the next lift

9) Calculate button

(11)

10) Calculated load and estimated 1RM based

on inputted data

11) Velocity zone, and stop information for inputted relative load

Figure 3. Components of a MATLAB WebApp to create and use load velocity profiles (LVP) to dictate training load. Available at https://matlabwebapps.port.ac.uk/webapps/home/ 


\subsubsection{Guidelines}

A number of approaches to establishing an LVP are available and each may offer certain advantages. The authors present one approach used by Dorrell, Moore and Gee (5). During all lifts, the load and mean concentric velocity should be measured for all repetitions. To obtain a more robust measure of velocity, the average of the repetitions completed at each load should be used to create the profile. The profiling procedure presented here (Figure 4) is an adaptation of the 1RM guidelines established by the National Strength and Conditioning Association (23).

- Initial load should be set to approximately $30 \%$ estimated $1 \mathrm{RM}$, or as close as equipment allows - starting at such a low load creates a more complete LVP even if the profile is not intended to be used at such low loads.

- Lifts should be completed at incremental loads of $5 \%$ estimated 1RM following completion of successful repetitions - having velocity data at this many relative loads allows the LVP to be modelled more accurately.

- For light loads $(\leq 50 \%$ estimated 1RM) participants should complete three repetitions, decreasing to two repetitions for medium loads (55-80\% estimated 1RM), and a single repetition for high loads ( $\geq 85 \%$ estimated $1 \mathrm{RM}$ ) - by only completing a low number of repetitions for each load the risk of fatiguing the athlete is reduced.

- Once an athlete has completed a repetition at approximately $95 \%$ of their estimated 1RM the load increments should be adjusted to ensure the athlete's true 1RM is reached and recorded.

- Finally, relative load (\%1RM) of each lift should be calculated and plotted against velocity before a linear line of best fit or a second order polynomial is fit to the data this is achievable in a variety of software (e.g. Microsoft Excel) however, the provided online application can perform these tasks.

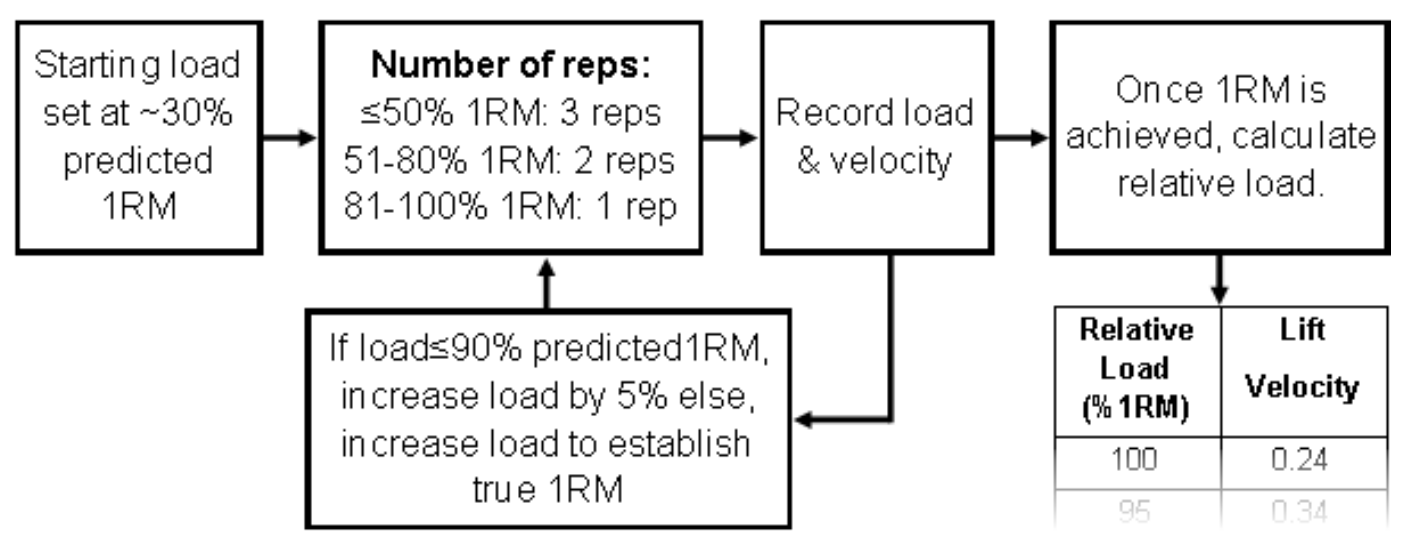

Figure 4. Flow diagram of a method of profiling an athlete to establish a load-velocity profile 
The online application is capable of creating an LVP from included group velocity data or by importing a Microsoft Excel document. Preprogramed group data is available by selecting the desired movement from the drop down menu and clicking the "Group Profile" button (Component 4 in Figure 3). The athlete details of the group data is provided in Table 1. To load external data the "Load Profile" should be clicked (Component 5 in Figure 3) and a Microsoft Excel document opened. The spreadsheet is required to be in a certain configuration with relative load in the first column and the corresponding velocity in the second column. An example profile is provided as part of this article (Supplementary Material 2). An additional step that is required when creating the LVP is to set the level of error that is to be accounted for in subsequent calculations. The advice of the authors is to use $68 \%$ for individual profiles and $34 \%$ for group profiles (Component 6 in Figure 3).

Table 1. Mean \pm SD of athlete information for the group load velocity profiles for the deadlift and strict overhead press (7), and squat (5) included in the online application.

\begin{tabular}{lcc}
\hline & Deadlift and Strict Press & Squat \\
\hline Number of Participants & 16 & 19 \\
Age $($ years $)$ & $22.8 \pm 4.5$ & $23.6 \pm 3.7$ \\
Height $(\mathrm{m})$ & $1.80 \pm 0.06$ & $1.83 \pm 0.05$ \\
Mass $(\mathrm{kg})$ & $89.3 \pm 13.3$ & $92.2 \pm 8.7$ \\
Squat $1 R M(\mathrm{~kg})$ & $147.8 \pm 25.0$ & $150.7 \pm 23.7$ \\
Deadlift $1 R M(\mathrm{~kg})$ & $176.4 \pm 31.4$ & $\mathrm{NA}$ \\
Strict Press $1 R M(\mathrm{~kg})$ & $64.6 \pm 8.5$ & $\mathrm{NA}$ \\
\hline
\end{tabular}

\subsection{Use of the Profile}

\subsubsection{Background}

There are many uses of the LVP however, the method used by Dorrell, Moore and Gee (5) by which prescribed load can be regulated is described here. Regardless of whether a group or individual LVP is used, a mathematical equation is created that describes the relationship between the mean concentric velocity of a lift and the relative load that it represents. Additionally, by calculating the confidence limits (the boundaries of the data) of the LVP, the variation of an athlete's velocity at a set relative load can also be taken into account. If the assumption that regardless of changes in absolute strength the LVP remains constant, the relative load an athlete has lifted and therefore their current $1 \mathrm{RM}$ can be calculated. The authors of this article recommend a number of steps to conduct this process. $A$ worked example is presented in Figure 5.

1. To account for the variation and minimise the risk of overestimating an athletes $1 R M$, the equation of the upper boundary of the LVP is solved for the measured lift velocity to provide an estimate of the relative load.

2. Using the estimated relative load, the athletes 1RM can be calculated by dividing the load by the relative load as a decimal (e.g. $80 \%=0.80$ ).

3. Using this estimated $1 \mathrm{RM}$, the load of the next set can be calculated taking into account the current strength of the athlete. 
a)

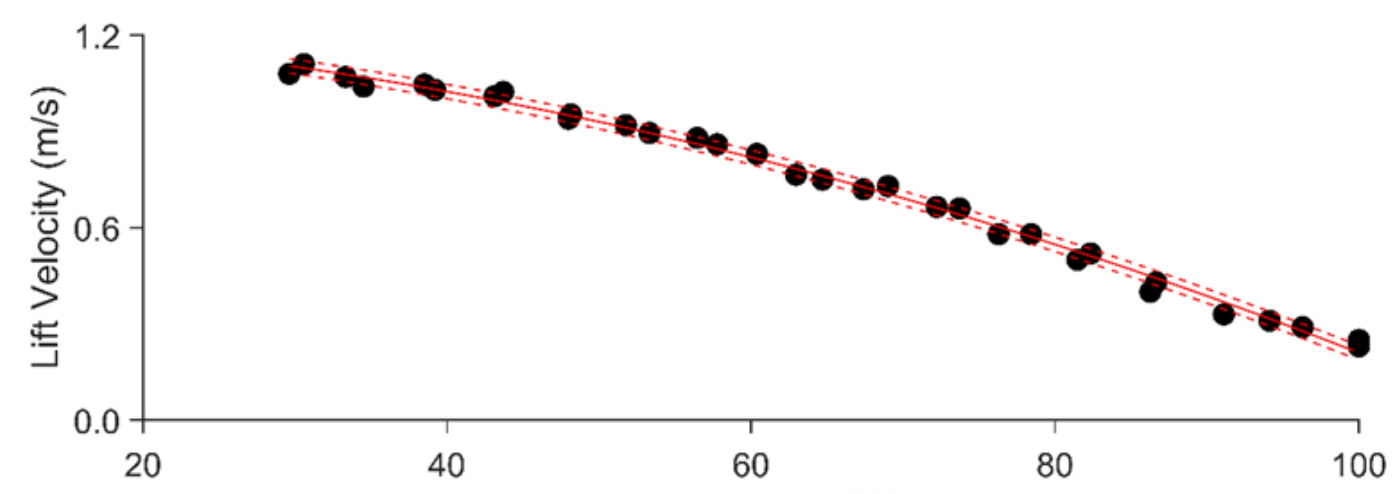

b)

Relative Load (\%)

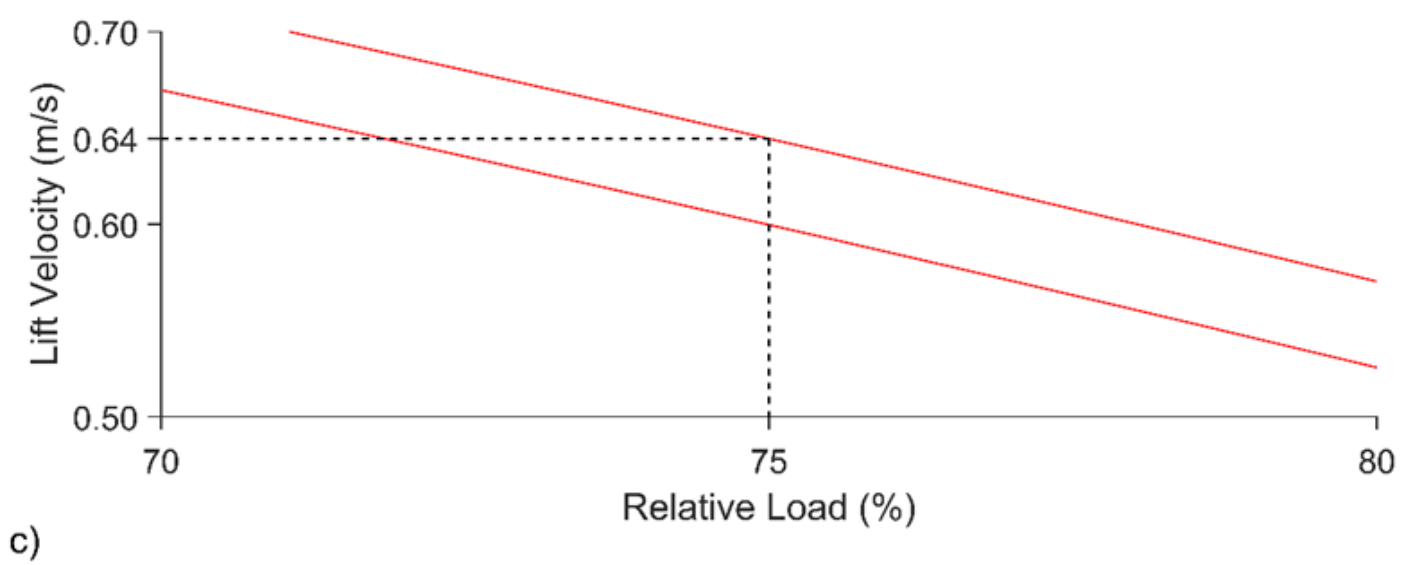

1. An athlete performs a $\mathbf{1 0 0} \mathbf{~ k g}$ back squat with a mean concentric velocity of $0.64 \mathrm{~m} / \mathrm{s}$.

2. Using the athlete's load velocity profile and velocity of the lift, $\mathbf{1 0 0} \mathbf{~ k g}$ is shown to be $\mathbf{7 5 \%}$ of the athlete's $1 \mathrm{RM}(\mathrm{b})$.

3. We can then predict that athlete's acute $1 \mathrm{RM}: 75 \%=100 \mathrm{~kg} ; \mathbf{1 0 0} \%=$ $133 \mathrm{~kg}$.

4. The next programmed relative load is $\mathbf{8 0} \%$ so the athlete should lift $106 \mathrm{~kg}$.

Figure 5. a) A load velocity profile calculated from one-repetition maximum testing, b) how the relative load of a lift can be determined using its velocity, and c) a worked example of determining the correct load for a preprogrammed relative load. 
The use of the described method to predict an athlete's 1RM would allow a practitioner to manipulate the load of each set to ensure that an athlete is lifting the correct absolute load relating to the prescribed relative load. Despite this attractive prospect, and the evidence to support such approaches $(5,7)$, a number of barriers limit its potential use in applied settings. Some of these barriers are the need to conduct a number of computational steps including creating the LVP by fitting a polynomial, solving the equation of the line using the measured velocity, and finally calculating the athlete's acute 1RM. To address these limitations, simple guidelines are provided on how to use an LVP to manipulate load, including how the provided online application can be used within applied practice.

\subsubsection{Guidelines}

Once an LVP has been established the process for estimating an athlete's acute 1RM and therefore allowing adjustment of absolute load is the same, irrespective of the LVP used. To allow for the estimation of an athlete's acute 1RM, the load and

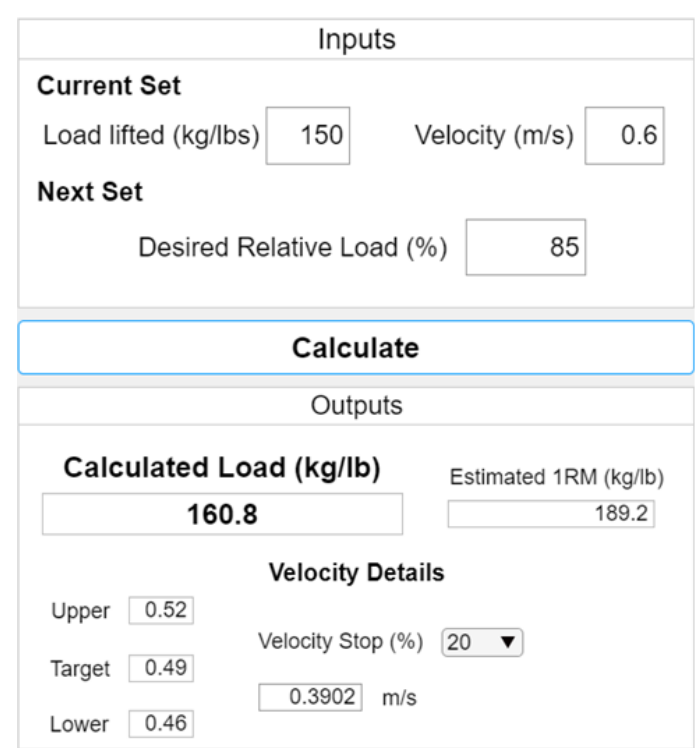

velocity of a repetition is required. In practice, this would most likely be the final warm up set or the previous working set. This information in combination with the desired relative load can be inputted into the online application (Components $7 \& 8$ in Figure 3 ) to calculate the absolute load of the next set (Figure 6). This process can be repeated after each working set meaning set-by-set auto-regulation can be achieved. It should be noted that the inputted data could be of any relative load that is within the assessed LVP boundaries (i.e. greater than $30 \% 1 \mathrm{RM}$ ). An example of how the described approach to autoregulation could be included into practice is by regulating the load of the main compound lift. For example, by loading the group squat LVP on a tablet or laptop running the application, athletes could input their load and velocity of a previous set, and the programmed relative load of the next set to inform them what absolute load should be on the bar. The use of a group profile would allow multiple athletes to use the application together automating the decisions about what load should be lifted, therefore reducing the practitioner burden, and maximising the effectiveness of the designed programme.

\begin{tabular}{l}
\begin{tabular}{c} 
Input Data \\
$\begin{array}{c}\text { To inform the next set, data from a previous set } \\
\text { completed on the same day is needed } \\
\text { (e.g. warm up or last working set) }\end{array}$ \\
\begin{tabular}{|l|}
\hline INPUT LOAD AND VELOCITY OF PREVIOUS SET \\
\&
\end{tabular} \\
INPUT RELATIVE LOAD (\%) OF NEXT SET \\
\hline "Calculate" \\
Outputs \\
-Calculated load - this is the load of the next set \\
•-Estimated 1RM - estimated athlete acute 1RM \\
-Velocity details - the velocity zone of the inputted \\
relative load and associated percentage drop off \\
threshold
\end{tabular} \\
\hline
\end{tabular}

Figure 6. Process of calculating absolute load based on a load velocity profile, lift velocity, and desired relative load 


\subsection{Considerations for Application to Practice}

The need for more sophisticated autoregulation methods is driven by the need for S\&C practitioners to maximise the effectiveness of their prescribed programs by optimising training variables. This article has described how VBT and the use of LVPs can provide an approach that allows for set-by-set regulation based on previously established LVPs. An online application and guidelines for profiling are provided.

The worth of the described approach is how it is able to progress or regress prescribed load based on the athlete's acute strength. As previously discussed, the LVP appears to be consistent for an athlete despite changes in absolute strength. Meaning that even if an athlete increases (or decreases) their 1RM by $5 \mathrm{~kg}$, they will still lift $80 \%$ of their $1 R M$ at the same velocity. This apparent trait of an LVP means that the presented method of autoregulation is able to manipulate the load lifted to ensure the athlete is always lifting the prescribed relative load. Such an approach has been found to be more effective than traditional percentage based approaches (7), with individual LVPs offering even more benefit (5). If an LVP can be established, such as those provided in the online application, the described approach may be a solution to the problem of how to regulate load. However, any new approaches will be associated with additional athlete and practitioner burden.

The online application included in this article was designed to minimise the negative effects the computational steps of VBT would have on a typical resistance training session. Once the LVP has been loaded into the online application, the process of calculating the next load only requires the input of three numbers. The calculations described in Section 2.2.1 are then completed instantly on clicking "Calculate". Additionally, as only the first repetition of a set is required, the process could be completed before the athlete has finished their set. These characteristics of the described VBT method may still make it unsuitable in certain S\&C environments, however it is hoped that this article has made the approach more accessible to practitioners.

To conclude VBT and the use of LVPs may offer an objective approach to autoregulation that is able to progress and regress absolute load based on an estimation of an athlete's acute strength. Factors such physiological adaptations, readiness to train, and fatigue would all theoretically result in changes in the athlete's 1RM on that day however, as the LVP states that the relationship between relative load and velocity is consistent the described approach may be able to account for these factors. It is accepted that there are barriers to the implementation of VBT as method of auto-regulation but the provided guidelines and online application is intended to minimise these. 


\section{References}

1. Balsalobre-Fernández C, García-Ramos A, and Jiménez-Reyes $P$. Load-velocity profiling in the military press exercise: effects of gender and training. Int J Sports Sci Coach 13:743-750, 2017. https://doi.org/10.1177/1747954117738243

2. Balsalobre-Fernández C, Marchante D, MuñozLópez $M$, and Jiménez SL. Validity and reliability of a novel iPhone app for the measurement of barbell velocity and $1 \mathrm{RM}$ on the bench-press exercise. $J$ Sports Sci 36:64-70, 2018. https://doi.org/10.1080/02640414.2017.1280610

3. Banyard HG, Nosaka K, and Haff GG. Reliability and validity of the load-velocity relationship to predict the 1RM back squat. J Strength Cond Res 31:18971904, 2017. https://doi.org/10.1519/jsc.0000000000001657

4. Banyard HG, Tufano JJ, Delgado J, Thompson SW, and Nosaka K. Comparison of the effects of velocity-based training methods and traditional 1RM-percent-based training prescription on acute kinetic and kinematic variables. Int J Sports Physiol Perform 14:246-255, 2019. https://doi.org/10.1123/ijspp.2018-0147

5. Dorrell HF, Moore JM, and Gee TI. Comparison of individual and group-based load-velocity profiling as a means to dictate training load over a six-week strength and power intervention, J Strength Cond Res 34:e4, 2020. https://journals.Iww.com/nscajscr/Fulltext/2020/01000/Abstracts.33.aspx

6. Dorrell HF, Moore JM, Smith MF, and Gee TI. Validity and reliability of a linear positional transducer across commonly practised resistance training exercises. J Sports Sci 37:67-73, 2019. https://doi.org/10.1080/02640414.2018.1482588

7. Dorrell HF, Smith MF, and Gee TI. Comparison of velocity-based and traditional percentage-based loading methods on maximal strength and power adaptations. J Strength Cond Res, 2019. https://doi.org/10.1519/JSC.0000000000003089

8. Fry AC. The role of resistance exercise intensity on muscle fibre adaptations. Sports Med 34:663-679, 2004. 200434100-00004

9. Garcia-Ramos A, Torrejon A, Feriche B, MoralesArtacho AJ, Perez-Castilla A, Padial P, and Haff GG. Prediction of the maximum number of repetitions and repetitions in reserve from barbell velocity. Int J Sports Physiol Perform 13:353-359, 2018. https://doi.org/10.1123/ijspp.2017-0302
10. Gonzalez-Badillo JJ and Sanchez-Medina L. Movement velocity as a measure of loading intensity in resistance training. Int J Sports Med 31:347-352, 2010. https://doi.org/10.1055/s-0030$\underline{1248333}$

11. Hackett DA, Cobley SP, Davies TB, Michael SW, and Halaki M. Accuracy in estimating repetitions to failure during resistance exercise. J Strength Cond Res 31:2162-2168, 2017. https://doi.org/10.1519/JSC.0000000000001683

12. Hackett DA, Johnson NA, Halaki M, and Chow CM. A novel scale to assess resistance-exercise effort. J Sports Sci 30:1405-1413, 2012. https://doi.org/10.1080/02640414.2012.710757

13. Helms ER, Byrnes RK, Cooke DM, Haischer MH, Carzoli JP, Johnson TK, Cross MR, Cronin JB, Storey $A G$, and Zourdos MC. RPE vs. percentage 1RM loading in periodized programs matched for sets and repetitions. Front Physiol 9:247-247, 2018. https://doi.org/10.3389/fphys.2018.00247

14. Helms ER, Cronin J, Storey A, and Zourdos MC. Application of the repetitions in reserve-based rating of perceived exertion scale for resistance training. Strength Cond J 38:42-49, 2016. https://doi.org/10.1519/SSC.0000000000000218

15. Jovanović $M$ and Flanagan EP. Researched applications of velocity based strength training. $J$ Aust Strength Cond 22:58-69, 2014.

16. Kraemer WJ and Ratamess NA. Fundamentals of resistance training: progression and exercise prescription. Med Sci Sports Exerc 36:674-688, 2004.

https://doi.org/10.1249/01.mss.0000121945.3663 $\underline{5.61}$

17. Lake J, Naworynsky D, Duncan F, and Jackson M. Comparison of different minimal velocity thresholds to establish deadlift one repetition maximum. Sports 5:70, 2017 https://doi.org/10.3390/sports5030070

18. Ruf L, Chery C, and Taylor KL. Validity and reliability of the load-velocity relationship to predict the onerepetition maximum in deadlift. J Strength Cond Res 32:681-689, 2018. https://doi.org/10.1519/JSC.0000000000002369

19. Sanchez-Medina L and Gonzalez-Badillo JJ. Velocity loss as an indicator of neuromuscular fatigue during resistance training. Med Sci Sports Exerc 43:17251734 2011. https://doi.org/10.1249/MSS.0b013e318213f880

20. Sanchez-Medina L, Gonzalez-Badillo JJ, Perez CE, and Pallares JG. Velocity- and power-load relationships of the bench pull vs. bench press 
exercises. Int J Sports Med 35:209-216, 2014.

https://doi.org/10.1055/s-0033-1351252

21. Schoenfeld BJ. The mechanisms of muscle hypertrophy and their application to resistance training. J Strength Cond Res 24:2857-2872, 2010. https://doi.org/10.1519/JSC.0b013e3181e840f3

22. Seo DI, Kim E, Fahs CA, Rossow L, Young K, Ferguson SL, Thiebaud R, Sherk VD, Loenneke JP, Kim D, Lee MK, Choi KH, Bemben DA, Bemben MG, and So WY. Reliability of the one-repetition maximum test based on muscle group and gender. J Sports Sci Med 11:221-225, 2012.

23. Sheppard JM and Triplett NT. Program design for resistance training, in: Essentials of Strength Training and Conditioning. GG Haff, NT Triplett, eds. Champaign, IL: Human Kinetics, 2015.

24. Stone $\mathrm{MH}$, Potteiger JA, Pierce $\mathrm{KC}$, Proulx $\mathrm{CM}$, O'Bryant HS, Johnson RL, and Stone ME. Comparison of the effects of three different weight-training programs on the one repetition maximum squat. J Strength Cond Res 14:332-337, 2000. http://dx.doi.org/10.1519\%2F00124278200008000-00015

25. Verdijk LB, van Loon L, Meijer K, and Savelberg HH. One-repetition maximum strength test represents a valid means to assess leg strength in vivo in humans. J Sports Sci 27:59-68, 2009. https://doi.org/10.1080/02640410802428089

26. Williams S, Trewartha G, Cross MJ, Kemp SPT, and Stokes KA. Monitoring what matters: a systematic process for selecting training-load measures. Int $\mathrm{J}$ Sports Physiol Perform 12:S2101-S2106, 2017. https://doi.org/10.1123/ijspp.2016-0337

27. Zourdos MC, Klemp A, Dolan C, Quiles JM, Schau $K A$, Jo E, Helms E, Esgro B, Duncan S, Garcia Merino $S$, and Blanco $R$. Novel resistance training-specific rating of perceived exertion scale measuring repetitions in reserve. J Strength Cond Res 30:267275,

2016.

https://doi.org/10.1519/JSC.0000000000001049 